\title{
Gastrointestinal cancers - still many questions to be resolved
}

\section{E. Wöll}

St. Vinzenz Hospital Zams, Zams, Austria

Received 8 April 2011; accepted 19 April 2011

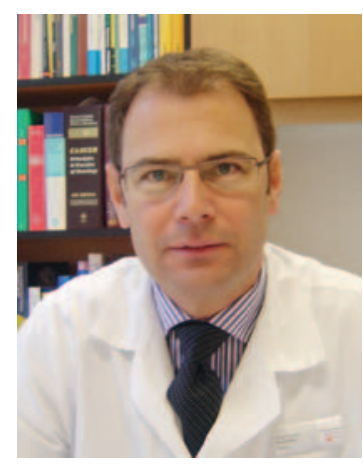

Ewald Wöll
Treatment of gastrointestinal neoplasias has changed substantially during the last decade. Nihilism and poor response rates have been replaced by aggressive treatment strategies and even curative approaches in metastatic colorectal cancer for example. A major achievement is the multidisciplinary treatment - lead by tumour boards - enabling optimal treatment sequences and utilization of different treatment modalities. Increase in response rates and prolongation of survival is also achieved by the use of novel cytostatic drugs and implementation of so called "targeted therapies".

In spite of these undoubtedly positive developments many questions still remain unanswered and implementation of novel treatment strategies is not uniformly successful. Furthermore predictive markers for targeted therapies are warranted but preclinical and clinical data however are not conclusive.

This issue of memo addresses some of the controversial topics and focuses on treatment standards in gastrointestinal cancers.

The first part covers early stage colorectal cancer. Adjuvant chemotherapy in stage III and stage II disease is discussed by Thaler and Resch [1] as well as controversial aspects of adjuvant or neoadjuvant chemoradiation in rectal cancer by Hoffmann et al. [2].

In the second part several reviews shed light on different aspects of advanced colorectal cancer. Recent developments and future perspectives in medical treatment of stage IV colorectal cancer are discussed by Hacker et al. [3] as well as optimal sequencing of surgery and chemo-/immunotherapy by Zitt [4] and the option of local radiofrequency ablation as dealt with by Bale and Widmann [5].

In the last part oesophageal carcinoma, gastric cancer, hepatocellular carcinoma and cholangiocellular carcinoma are covered by Gabriel [6], Ruhstaller and Stahl [7], Lordick et al. [8] and Köberle and Samaras [9]. In some of these entities treatment standards have been changed substantially. In inoperable gastric cancer HER 2 positivity serves as a predictive marker for trastuzumab in combination with chemotherapy. In hepatocellular carcinoma and cholangiocellular carcinoma treatment in inoperable disease could be improved substantially.

Optimal treatment however includes aggressive side effect management. Therefore one review addresses special aspects of side effect management in gastrointestinal cancers [10].

I am very grateful to all authors for their excellent reviews and the timely delivery and I appreciate the willingness to comment controversial topics.

\section{Conflict of interest}

The author declares that there is no conflict of interest.

\section{References}

[1] Thaler J, Resch G. Adjuvant therapy in colon carcinoma stages II and III. memo, 4(2): 86-89, 2011.

[2] Hoffmann W, Lordick F, Becker-Schiebe M. (Neo-) Adjuvant Radio Chemotherapy in stage II/III Rectal cancer. memo, 4(2): 90-93, 2011.

[3] Hacker UT, Coutelle O, Hallek M. Recent developments and future persepectives in systemic treatment of metastatic colorectal cancer memo, 4(2): 75-78, 2011.

[4] Zitt M, Bowel first? simultaneous resection? Liver First? Treatment options in patients with colorectal cancer and resectable synchronous liver metastases. memo, 4(2): 79-81, 2011.

[5] Bale R, Widmann G. Can radiofrequency ablation replace liver resection? memo, 4(2): 82-85, 2011.

[6] Gabriel M. Contribution of PET-CT in the management of GI-cancer. memo, 4(2): 102-104, 2011.

[7] Ruhstaller T, Stahl M. Current treatment strategies of esophageal carcinoma. memo, 4(2): 66-70, 2011.

[8] Lordick F, Schumacher G, Becker-Schiebe M. Gastric Cancer. memo, 4(2): 71-74, 2011

[9] Köberle D, Samaras P. Recent advances and future challenges in the treatment of hepatocellular and biliary tract carcinomas. memo, 4(2): 94-97, 2011

[10] Becker-Schiebe M, Lordick F, Hoffmann W. Side-effect management during. treatment of GI-cancers. memo, 4(2): 98-101, 2011. 